\title{
A Method for Computing the Iwasawa $\lambda$-Invariant
}

\author{
By R. Ernvall and T. Metsänkylä
}

\begin{abstract}
We present a method for computing the minus-part of the Iwasawa $\lambda$-invariant of an Abelian field $K$. Applying this method, we have computed $\lambda^{-}$for several odd primes $p$ when $K$ runs through a large number of quadratic extensions of the $p$ th cyclotomic field. A report on these computations and an analysis of the results is included.
\end{abstract}

1. Introduction. Let $K$ be an Abelian field, i.e., a finite Abelian extension of $\mathbf{Q}$. For a prime $p>2$, consider the cyclotomic $\mathbf{Z}_{p}$-extension $K_{\infty}$ of $K$. Let $K_{n}(n \geqslant 0)$ denote the intermediate field of $K_{\infty} / K$ which is cyclic of degree $p^{n}$ over $K$. The $p$-part of the class number of $K_{n}$ equals $p^{\lambda n+\nu}$, for all sufficiently large $n$, where $\lambda=\lambda(p)$ and $\nu=\nu(p)$ are integral constants, $\lambda \geqslant 0$. Call $\lambda$ the Iwasawa $\lambda$ invariant of $K$ and write $\lambda=\lambda^{+}+\lambda^{-}$, where $\lambda^{+}$is the corresponding invariant of the maximal real subfield of $K$. In this paper we present a method for computing $\lambda^{-}$, developed by the second author, and report on computer calculations by the first author, performed by this method.

If the conductor $f_{K}$ of the field $K$ is divisible by $p^{2}$, then $K$ has a subfield $L$ such that $p^{2}+f_{L}$ and the cyclotomic $\mathbf{Z}_{p}$-extension of $L$ equals $K_{\infty}$. Hence we assume, without loss of generality, that $p^{2}+f_{K}$. Denote by $\mathrm{Ch}(K)$ the character group of $K$. It is known that $\lambda^{-}$decomposes as

$$
\lambda^{-}=\sum_{\chi \in X} \lambda_{\chi}
$$

with

$$
X=X(K)=\left\{\chi \in \operatorname{Ch}(K): \chi(-1)=-1, \chi \neq \omega^{-1}\right\},
$$

where $\omega$ denotes the Teichmüller character $\bmod p$ and $\lambda_{\chi}$ is the $\lambda$-invariant of the Iwasawa power series representing the $p$-adic $L$-function $L_{p}(s, \chi \omega)$.

Thus, the computation of $\lambda^{-}$is reduced to the determination of the components $\lambda_{\chi}$. This will be done in two steps: We first relate $\lambda_{\chi}$ to the $p$-orders of certain generalized Bernoulli numbers and then show how to determine these $p$-orders by means of a series of character sum congruences. As an application we consider the fields $\mathbf{Q}\left(\sqrt{m}, \zeta_{p}\right)$, where $m$ is an integer prime to $p$ and $\zeta_{p}$ denotes a primitive $p$ th root of 1 . In this case the congruences in question are simply rational congruences $\bmod p$.

Received September 5, 1986.

1980 Mathematics Subject Classification (1985 Revision). Primary 11R23, 11R29, 11S40, 11Y40, 11-()4 
The computational part of our work consists of the determination of $\lambda^{-}$for quite a large collection of fields $\mathbf{Q}\left(\sqrt{m}, \zeta_{p}\right)$, chosen so that either $p$ or $|m|$ is small. More precisely, we computed for these fields the components $\lambda_{\chi}$ with $\chi=\theta_{m} \omega^{t}$, where $\theta_{m}$ is the quadratic character of the field $\mathbf{Q}(\sqrt{m})$; this is sufficient since the $\lambda^{-}$invariants of the cyclotomic subfields $\mathbf{Q}\left(\zeta_{p}\right)$ are known. Our results also give the $\lambda$-invariant of $\mathbf{Q}(\sqrt{m})$ for the negative $m$ in the range under consideration.

There are previous numerical results about $\lambda^{-}$in [1], [2], [3], [4], [6]. These concern mainly quadratic fields and the fields $\mathbf{Q}\left(\sqrt{-1}, \zeta_{p}\right)$ and $\mathbf{Q}\left(\sqrt{-3}, \zeta_{p}\right)$, and in all cases the decomposition of $\lambda^{-}$is simple in the sense that either there is but one positive component $\lambda_{\chi}$, or all the positive components are equal to 1 . In the present results this is no longer the case.

A detailed description of our computations appears in Sections 7-9.

2. On $p$-Adic $L$-Functions. For the theory of this section, the reader is referred to Washington's book [11], in particular to Sections 5.2 and 7.2.

We fix an embedding of the field of algebraic numbers in an algebraic closure $\Omega_{p}$ of $\mathbf{Q}_{p}$, the field of $p$-adic numbers. Denote by ord ${ }_{p}$ the $p$-adic valuation on $\Omega_{p}$, normalized so that ord $p(p)=1$.

Let $\chi$ be a character in $X(K)$ (all characters are assumed primitive). Since the conductor $f_{\chi}$ of $\chi$ divides $f_{K}$, it is not divisible by $p^{2}$; we say that $\chi$ is of the "first kind". Put

$$
f_{\chi}=d \text { or } d p \quad \text { with }(d, p)=1 \text {. }
$$

As in the introduction, let $K_{n}$ denote the $n$th layer of the $\mathbf{Z}_{p}$-extension $K_{\infty} / K$ $(n \geqslant 0)$. The character group of $K_{n}$ is of the form $\operatorname{Ch}(K) \times\left\langle\pi_{n}\right\rangle$, where $\pi_{n}$ is a character with order $p^{n}$ and conductor $p^{n+1}$ (or 1 , if $n=0$ ); $\pi_{n}$ is called a character of the "second kind".

Now consider the $p$-adic $L$-function $L_{p}(s, \psi)$ for the (nonprincipal) character $\psi=\chi \omega \pi_{n}$. This function is defined in $\Omega_{p}$ in a neighborhood of 1 containing $\mathbf{Z}_{p}$, the $p$-adic integers, and it has the fundamental property that

$$
L_{p}(1-k, \psi)=-\left(1-\psi_{k}(p) p^{k-1}\right) B^{k}\left(\psi_{k}\right) / k \quad(k \geqslant 1),
$$

where $\psi_{k}=\psi \omega^{-k}$ and $B^{k}\left(\psi_{k}\right)$ stands for the $k$ th generalized Bernoulli number attached to the character $\psi_{k}$.

Denote by $\mathbf{Q}_{p}(\chi)$ the extension of $\mathbf{Q}_{p}$ generated by the values of $\chi$. Iwasawa's theory of $p$-adic $L$-functions asserts that there exists a power series

$$
f(x, \chi \omega)=\sum_{j=0}^{\infty} a_{j} x^{j}
$$

whose coefficients $a_{j}=a_{j}(\chi)$ are integers of $\mathbf{Q}_{p}(\chi)$, such that

$$
L_{p}\left(s, \chi \omega \pi_{n}\right)=f\left(\frac{(1+d p)^{s}}{\pi_{n}(1+d p)}-1, \chi \omega\right) .
$$

According to the Ferrero-Washington theorem, the power series $f(x, \chi \omega)$ has $\mu=0$, in other words, there is an index $j$ for which ord ${ }_{p}\left(a_{j}\right)=0$. The least such $j$ is called the $\lambda$-invariant (or Weierstrass degree) of $f(x, \chi \omega)$. This is the number $\lambda_{\chi}$ introduced in Section 1. 
3. The $p$-Orders of Generalized Bernoulli Numbers. Let us decompose $\chi$ as

$$
\chi=\theta \omega^{t-1} \quad \text { with } f_{\theta}=d(\geqslant 1), \quad 1 \leqslant t \leqslant p-1 .
$$

In this section we obtain a relation between $\lambda_{\chi}$ and the $p$-order of $B^{t}\left(\theta \pi_{n}\right)$.

For a fixed $n \geqslant 1$, put

$$
\alpha_{k}=\frac{(1+d p)^{1-k}}{\pi_{n}(1+d p)}-1 \quad(k \geqslant 1) .
$$

It follows from (3) and (1) that, for all $t=1, \ldots, p-1$,

$$
\begin{aligned}
f\left(\alpha_{t}, \theta \omega^{t}\right) & =L_{p}\left(1-t, \theta \omega^{t} \pi_{n}\right) \\
& =-\left(1-\left(\theta \pi_{n}\right)(p) p^{t-1}\right) B^{t}\left(\theta \pi_{n}\right) / t=-B^{t}\left(\theta \pi_{n}\right) / t
\end{aligned}
$$

By using this result we prove the following proposition in which $\phi$ denotes Euler's totient function and $e$ is the ramification index of $\mathbf{Q}_{p}(\theta) / \mathbf{Q}_{p}$.

Proposition 1. Let $n \geqslant 1$ and $1 \leqslant t \leqslant p-1$. We have

$$
\begin{array}{ll}
\operatorname{ord}_{p}\left(B^{t}\left(\theta \pi_{n}\right)\right)=\lambda_{\chi} / \phi\left(p^{n}\right)<1 / e & \text { if } \lambda_{\chi}<\phi\left(p^{n}\right) / e, \\
\operatorname{ord}_{p}\left(B^{t}\left(\theta \pi_{n}\right)\right) \geqslant 1 / e & \text { if } \lambda_{\chi} \geqslant \phi\left(p^{n}\right) / e .
\end{array}
$$

Proof. We evaluate the $p$-order of $f\left(\alpha_{t}, \theta \omega^{t}\right)=\sum_{j=0}^{\infty} a_{j} \alpha_{t}^{j}$.

By the definition of $\pi_{n}$, the number $\pi_{n}(1+d p)=\zeta$ is a primitive $p^{n}$ th root of 1 . Since

$$
\alpha_{t}=\frac{1-\zeta(1+d p)^{t-1}}{\zeta(1+d p)^{t-1}},
$$

we have ord ${ }_{p}\left(\alpha_{t}\right)=\operatorname{ord}_{p}(1-\zeta)=1 / \phi\left(p^{n}\right)$.

As to the $p$-orders of the coefficients $a_{j}$, observe that these are integers of $\mathbf{Q}_{p}(\chi)=\mathbf{Q}_{p}(\theta)$. Therefore, if $\operatorname{ord}_{p}\left(a_{j}\right)>0$ then $\operatorname{ord}_{p}\left(a_{j}\right) \geqslant 1 / e$.

Recalling the definition of $\lambda_{\chi}$ we now see that

$$
\begin{aligned}
\operatorname{ord}_{p}\left(a_{j} \alpha_{t}^{j}\right) & \geqslant 1 / e & & \text { for } 0 \leqslant j \leqslant \lambda_{\chi}-1, \\
& =j \operatorname{jord}_{p}\left(\alpha_{t}\right)=\lambda_{\chi} / \phi\left(p^{n}\right) & & \text { for } j=\lambda_{\chi} \\
& \geqslant j \operatorname{jord}_{p}\left(\alpha_{t}\right)>\lambda_{\chi} / \phi\left(p^{n}\right) & & \text { for } j>\lambda_{\chi} .
\end{aligned}
$$

Consequently, if $\lambda_{\chi}<\phi\left(p^{n}\right) / e$, then

$$
\operatorname{ord}_{p}\left(f\left(\alpha_{t}, \theta \omega^{t}\right)\right)=\lambda_{\chi} / \phi\left(p^{n}\right),
$$

while otherwise this $p$-order is at least $1 / e$. Hence the result.

Proposition 1 gives us the value of $\lambda_{\chi}$, once we know ord ${ }_{p}\left(B^{t}\left(\theta \pi_{n}\right)\right)$ for a sufficiently large $n$. For later purposes it is convenient to reformulate this proposition, actually in a bit weaker form, as follows.

Note that the congruence $\alpha \equiv \beta\left(\bmod p^{r}\right)$ in $\Omega_{p}$ means that $\operatorname{ord}_{p}(\alpha-\beta) \geqslant r$.

Proposition 2. Let $n \geqslant 1$ and $1 \leqslant t \leqslant p-1$. Assume that $h \in \mathbf{Z}, 1 \leqslant h \leqslant$ $\phi\left(p^{n}\right) / e$. Then

$$
\lambda_{\chi} \geqslant h \quad \text { if and only if } \quad B^{t}\left(\theta \pi_{n}\right) \equiv 0\left(\bmod p^{h / \phi\left(p^{\prime \prime}\right)}\right) .
$$


Proof. Suppose that the above congruence holds. If $\lambda_{\chi}<\phi\left(p^{n}\right) / e$, then a comparison of this congruence with the first part of Proposition 1 shows that $\lambda_{\chi} \geqslant h$. If $\lambda_{\chi} \geqslant \phi\left(p^{n}\right) / e$, then the assertion follows directly from the assumption made about $h$.

To verify the converse, apply both parts of Proposition 1 separately.

Remark. Proposition 2 is of the same kind as the main result in the second author's paper [8]. This relates $\lambda_{\chi}$ to certain Kummer type congruences of $B^{k}(\theta)$, provided $\lambda_{x} \leqslant p-1$. Proposition 2 would enable one to replace the proof presented in [8] by a somewhat simpler proof.

4. Bernoulli Numbers and Character Sums. We now express the residue of $B^{t}\left(\theta \pi_{n}\right)$ modulo $p$ in terms of suitable character sums.

For any character $\psi$ with conductor $f$ we have, in the usual symbolic notation,

$$
B^{k}(\psi)=\frac{1}{f} \sum_{a=1}^{f} \psi(a)(f B+a-f)^{k} \quad(k \geqslant 0)
$$

(e.g., [7, p. 134]), where the $B^{m}$ denote ordinary Bernoulli numbers. On changing the summation variable $a$ into $f-a$ we obtain

$$
B^{k}(\psi)=\frac{(-1)^{k} \psi(-1)}{f} \sum_{a=1}^{f} \psi(a)(a-f B)^{k} .
$$

Let $\psi=\theta \pi_{n}$ with $n \geqslant 1$. Then $\psi(-1)=(-1)^{t}$ since the character $\chi=\theta \omega^{t-1}$ is odd and $\pi_{n}$, being of $p$-power order, is even. Hence we find that

$$
\begin{aligned}
B^{t}\left(\theta \pi_{n}\right) & =\frac{1}{d p^{n+1}} \sum_{a=1}^{d p^{n+1}}\left(\theta \pi_{n}\right)(a)\left(a-d p^{n+1} B\right)^{t} \\
& \equiv \frac{1}{d p^{n+1}} \sum_{a=1}^{d p^{n+1}}\left(\theta \pi_{n}\right)(a) a^{t}-t B^{1} \sum_{a=1}^{d p^{n+1}}\left(\theta \pi_{n}\right)(a) a^{t-1}(\bmod p) .
\end{aligned}
$$

The second sum of the last expression vanishes $\bmod p$, as can be verified again by the transformation $a \rightarrow d p^{n+1}-a$. Therefore,

$$
B^{t}\left(\theta \pi_{n}\right) \equiv \frac{1}{d p^{n+1}} \sum_{a=1}^{d p^{n+1}}\left(\theta \pi_{n}\right)(a) a^{t}(\bmod p) .
$$

From this result we derive the following congruence which is of the same type as the classical Voronoi congruence for ordinary Bernoulli numbers. We point out that the congruence (in a sharper form) has also been proved by Slavutskiı̆ [9, congr. (6)].

Proposition 3. Let $b$ be a positive rational integer with $(b, d p)=1$. Then

$$
\left(b^{t}-\left(\theta \pi_{n}\right)(b)^{-1}\right) B^{t}\left(\theta \pi_{n}\right) \equiv t b^{t-1} \sum_{a=1}^{d p^{n+1}}\left(\theta \pi_{n}\right)(a) a^{t-1}\left[\frac{b a}{d p^{n+1}}\right](\bmod p),
$$

where, as in the above, $n \geqslant 1$ and $1 \leqslant t \leqslant p-1$.

Proof. Put $\psi=\theta \pi_{n}$. Let $a$ and $b$ be positive rational integers prime to $d p$. Keeping $b$ fixed, we write

$$
b a=d p^{n+1}\left[\frac{b a}{d p^{n+1}}\right]+r_{a}, \quad 0<r_{a}<d p^{n+1} .
$$


On raising this equation to the $t$ th power and multiplying by $\psi(a)=\psi(b)^{-1} \psi\left(r_{a}\right)$, we get

$$
\psi(a) b^{t} a^{t} \equiv \psi(b)^{-1} \psi\left(r_{a}\right) r_{a}^{t}+\psi(a) t r_{a}^{t-1} d p^{n+1}\left[\frac{b a}{d p^{n+1}}\right]\left(\bmod p^{2 n+2}\right) .
$$

If $a$ runs through $1, \ldots, d p^{n+1}$, excepting those numbers for which $(a, d p)>1$, then so does $r_{a}$. Summing over $a$ we find that (observe that $\psi(a)=0$ if $\left.(a, d p)>1\right)$

$$
\left(b^{t}-\psi(b)^{-1}\right) \sum_{a=1}^{d p^{n+1}} \psi(a) a^{t} \equiv t d p^{n+1} \sum_{a=1}^{d p^{n+1}} \psi(a) r_{a}^{t-1}\left[\frac{b a}{d p^{n+1}}\right]\left(\bmod p^{2 n+2}\right) .
$$

Since $r_{a} \equiv b a\left(\bmod p^{n+1}\right)$, this result together with (4) yields the claimed congruence.

5. The Main Result. Every rational integer $a$ prime to $p$ has the following unique representation $\bmod p^{n+1}$ :

$$
a \equiv \omega(a)(1+p)^{v(a)}\left(\bmod p^{n+1}\right), \quad 0 \leqslant v(a)<p^{n} .
$$

For $b \in \mathbf{Z},(b, d p)=1$, put

$$
S_{n k}=S_{n k}(b)=\sum_{v(a)=k} \theta(a) a^{t-1}\left[\frac{b a}{d p^{n+1}}\right] \quad\left(k=0, \ldots, p^{n}-1\right),
$$

where the sum is extended over those numbers $a$ for which $1 \leqslant a<d p^{n+1},(a, d p)$ $=1$ and $v(a)=k$. Moreover, set

$$
T_{u}=T_{u}^{(n)}=\sum_{k=u}^{p^{n}-1}\left(\begin{array}{l}
k \\
u
\end{array}\right) S_{n k} \quad\left(u=0, \ldots, p^{n}-1\right)
$$

THEOREM. Let $\chi=\theta \omega^{t-1} \in X(K)$, where $f_{\theta}=d$ is prime to $p$ and $1 \leqslant t \leqslant p-1$. Let $b$ be a positive integer such that

$$
(b, d p)=1, \quad \theta(b) b^{t} \not \equiv 1(\bmod p)
$$

where $\mathfrak{p}$ is the maximal ideal of the ring of integers of $\mathbf{Q}_{p}(\theta)$. Denote by $e$ the ramification index of $\mathbf{Q}_{p}(\theta) / \mathbf{Q}_{p}$. Let $n \geqslant 1$ and let $h \in \mathbf{Z}, 1 \leqslant h \leqslant \phi\left(p^{n}\right) / e$. With the above notations,

$$
\lambda_{\chi} \geqslant h \quad \text { if and only if } \quad T_{0}^{(n)} \equiv T_{1}^{(n)} \equiv \cdots \equiv T_{h-1}^{(n)} \equiv 0(\bmod \mathfrak{p})
$$

Proof. Since the nonzero values of $\pi_{n}$ are $p^{n}$ th roots of 1 , we have $\pi_{n}(b) \equiv 1$ $(\bmod \mathfrak{p})$. Hence

$$
b^{t}-\left(\theta \pi_{n}\right)(b)^{-1} \not \equiv 0(\bmod \mathfrak{p}),
$$

and it follows from Propositions 2 and 3 that

$$
\lambda_{\chi} \geqslant h \quad \text { if and only if } \sum_{a=1}^{d p^{n+1}} \theta(a) \pi_{n}(a) a^{t-1}\left[\frac{b a}{d p^{n+1}}\right] \equiv 0\left(\bmod p^{h \kappa}\right),
$$

where $\kappa=1 / \phi\left(p^{n}\right)$.

For a fixed $n \geqslant 1$, write

$$
\pi_{n}(1+p)=1+\eta .
$$

Then we have ord $p(\eta)=\kappa$ and, by (5),

$$
\pi_{n}(a)=(1+\eta)^{v(a)} \text { for } p+a .
$$


Consequently,

$$
\sum_{a=1}^{d p^{n+1}} \theta(a) \pi_{n}(a) a^{t-1}\left[\frac{b a}{d p^{n+1}}\right]=\sum_{k=0}^{p^{n}-1}(1+\eta)^{k} S_{n k}=\sum_{u=0}^{p^{n}-1} T_{u} \eta^{u}
$$

and we are done, once the congruence

$$
\sum_{u=0}^{p^{n}-1} T_{u} \eta^{u} \equiv 0\left(\bmod p^{h \kappa}\right)
$$

is shown to be equivalent to

$$
T_{0} \equiv T_{1} \equiv \cdots \equiv T_{h-1} \equiv 0(\bmod \mathfrak{p}) \text {. }
$$

Suppose that the congruences (9) hold true. Then these congruences are satisfied $\bmod p^{1 / e}$ as well, and so $\bmod p^{h \kappa}$ since $1 / e \geqslant h / \phi\left(p^{n}\right)=h \kappa$. Moreover, $\eta^{u} \equiv 0$ $\left(\bmod p^{h \kappa}\right)$ whenever $u \geqslant h$. This proves (8). The converse implication is established with similar arguments by induction on $h$.

The above theorem enables us to determine $\lambda_{\chi}$, once the numbers $T_{u}^{(n)}$ modulo $\mathfrak{p}$ are known for a sufficiently large $n$. We state this more explicitly as follows.

Corollary. Put $z_{n}=\left[\phi\left(p^{n}\right) / e\right]$. With the notations of the theorem,

(i) if $T_{0}^{(n)} \equiv T_{1}^{(n)} \equiv \cdots \equiv T_{h-1}^{(n)} \equiv 0$ and $T_{h}^{(n)} \not \equiv 0(\bmod \mathfrak{p})$, where $0 \leqslant h \leqslant z_{n}-$ 1 , then $\lambda_{\chi}=h$;

(ii) if $T_{0}^{(n)} \equiv T_{1}^{(n)} \equiv \cdots \equiv T_{z_{n}-1}^{(n)} \equiv 0(\bmod \mathfrak{p})$, then $\lambda_{\chi} \geqslant z_{n}$.

6. A Special Case. Suppose that $\theta=\theta_{m}$ is the nontrivial character of the quadratic field $\mathbf{Q}(\sqrt{m})$, where $m$ is prime to $p$. Then the character $\chi=\theta \omega^{t-1}$ dealt with in the previous sections belongs to the character group of the field $\mathbf{Q}\left(\sqrt{m}, \zeta_{p}\right)$. Note that $f_{\theta}=d$ equals the absolute value of the discriminant of $\mathbf{Q}(\sqrt{m})$.

In this case, $\mathbf{Q}_{p}\left(\boldsymbol{\theta}_{m}\right)=\mathbf{Q}_{p}$, so that $e=1$ and $\mathfrak{p}=p \mathbf{Z}_{p}$. Hence we can determine $\lambda_{\chi}$, provided it does not exceed $p-2$, through the numbers $T_{u}=T_{u}^{(1)}$ as follows (see the corollary): $h$.

If $T_{0} \equiv T_{1} \equiv \cdots \equiv T_{h-1} \equiv 0, T_{h} \not \equiv 0(\bmod p)$, where $0 \leqslant h \leqslant p-2$, then $\lambda_{x}=$

If this criterion fails, then the computation of $\lambda_{\chi}$ requires passing to a higher level, i.e., computing $T_{u}^{(n)} \bmod p$ for a higher value of $n$.

Remark. As is seen from (5), working on a level $n$ involves computations with integers $\bmod p^{n+1}$. We point out that, for $n=1$, the congruence (5) can be written as

$$
a \equiv a^{p}(1+v(a) p)\left(\bmod p^{2}\right) .
$$

Thus, $v(a) \equiv-q_{a}(\bmod p)$, where $q_{a}$ denotes the Fermat quotient for $a$, defined by $q_{a} \equiv\left(a^{p-1}-1\right) / p(\bmod p), 0 \leqslant q_{a}<p$.

7. Numerical Results. Consider, for a moment, the case of the cyclotomic field $\mathbf{Q}\left(\zeta_{p}\right)$. Then $X=\left\{\omega, \omega^{3}, \ldots, \omega^{p-4}\right\}$ and it is known that

$$
\lambda_{\chi}>0 \quad \text { with } \chi=\omega^{t-1} \quad \text { if and only if } \quad B^{t} \equiv 0(\bmod p)
$$

$(t=2,4, \ldots, p-3)$. The values of $\lambda_{\chi}$ have been computed for $p<125000$ [10]; it has turned out that in this range every positive value of $\lambda_{\chi}$ equals 1 . So the $\lambda^{-}$-invariant of $\mathbf{Q}\left(\zeta_{p}\right)$, say $\lambda_{0}^{-}$, equals the index of irregularity of $p$, i.e., the number of irregular pairs $(p, t)$. Tables of irregular pairs can be found in many books, e.g., [11]. 
Now let us enlarge the field to $K=\mathbf{Q}\left(\sqrt{m}, \zeta_{p}\right)$ with $p+m$. Then the character set $X$ is enlarged by the characters $\theta_{m} \omega^{t-1}$ discussed in Section 6 . To be precise, we have

$$
\lambda^{-}=\lambda_{0}^{-}+\sum_{\chi} \lambda_{\chi}
$$

where the sum is extended over the characters

$$
\chi=\theta \omega^{t-1} \text { with } \begin{cases}t=2,4, \ldots, p-1 & \text { if } m>0 \\ t=1,3, \ldots, p-2 & \text { if } m<0\end{cases}
$$

$\boldsymbol{\theta}=\boldsymbol{\theta}_{\boldsymbol{m}}$ being the quadratic character of $\mathbf{Q}(\sqrt{m})$. If $m<0$, the component $\lambda_{\theta}$ is just the $\lambda$-invariant of this quadratic field.

The actual computations associated with the present work comprised the determination of $\lambda_{x}$ for the characters (10) when $p$ and $m$ range through the following values ( $m$ squarefree):

$$
\begin{aligned}
& p=3 \quad \text { and } \quad-3235 \leqslant m \leqslant 3454,{ }^{*} \\
& p=5 \quad \text { and } \quad-5000<m \leqslant 3147, \\
& p=7 \quad \text { and } \quad-3002 \leqslant m<1000, \\
& p=11 \quad \text { and } \quad-1000<m<500, \\
& 11<p<200 \quad \text { and } m=-7,-3,-2,-1,2,5 .
\end{aligned}
$$

The asterisk above indicates that for a few values of $m$ the computation was stopped at the result $\lambda_{\chi} \geqslant 6$ (see below).

The numerical material thus obtained contains about 22000 values of $\lambda_{\chi}$, some 6400 of them being positive. Samples from this material are exhibited in Tables 1 and 2 of the appendix. Table 1 presents the results for $p=5, m>0$, and Table 2 for $p<200, m=-1, \pm 2,-3,5,-7$. Note that every odd prime $p$ below 200 really appears in Table 2, i.e., to every $p$ there is at least one $m$ and $t$ such that $\lambda_{\chi}>0$ for $\chi=\theta_{m} \omega^{t-1}$.

For $p>3$, only few cases were found in which $\lambda_{\chi}>p-2$. These cases, which had to be settled on the level $n=2$, are listed here:

\begin{tabular}{c|c|c|c||c|c|c|c}
$p$ & $m$ & $t$ & $\lambda_{x}$ & $p$ & $m$ & $t$ & $\lambda_{x}$ \\
\hline 5 & 439 & 4 & 4 & 5 & -3178 & 1 & 4 \\
5 & 1427 & 4 & 4 & 5 & -3471 & 1 & 4 \\
5 & -311 & 1 & 4 & 5 & -3547 & 3 & 4 \\
5 & -761 & 1 & 4 & 5 & -3923 & 3 & 4 \\
5 & -966 & 1 & 4 & 5 & -4026 & 1 & 5 \\
5 & -2861 & 3 & 4 & 5 & -4774 & 1 & 4 \\
5 & -3081 & 1 & 4 & 7 & -1371 & 1 & 7
\end{tabular}

For $p=7$ it in fact turned out that $\lambda_{\chi}$ varies between 0 and 4 (assuming all values $0, \ldots, 4)$ except in the single case given above. For $p=11$ we have the maximum $\lambda_{x}=3$ for $m=-723, t=1$.

If $p=3$, then $\lambda_{\chi}>1(=p-2)$ in about a third of the cases. These could be settled on the level $n=2$ (i.e., $\lambda_{\chi} \leqslant 5$ ), except in six cases. In the latter cases the continuation of the procedure was given up since the values of $\lambda_{\chi}$ can be found in [6]; they are as follows:

$$
\begin{array}{ll}
\lambda_{\chi}=6 & \text { for } m=-239,-1022,-1427,-1777 \\
\lambda_{\chi}=7 & \text { for } m=-458 \\
\lambda_{\chi}=8 & \text { for } m=-2789
\end{array}
$$


An examination of the results shows that the values of $\lambda_{\chi}$ seem to be distributed in the expected way. For example, if we keep $p$ and $t$ fixed, $t \neq 1$, and let $m$ vary, then the number of cases with $\lambda_{\chi} \geqslant k$ (for $\chi=\theta_{m} \omega^{t-1}$ and $k \geqslant 0$ ) should be about a $p^{k}$ th part of the number of all $\lambda_{\chi}$; this corresponds to the natural hypothesis that the coefficients of the power series $f(x, \chi \omega)$ are randomly distributed mod $p$. In the following table, $N_{k}$ denotes the number of $\lambda_{x} \geqslant k$ in our range:

\begin{tabular}{c|c|c|c|r|r|c|c|c|c|c|c}
$p$ & $t$ & $N_{0}$ & $N_{1}$ & $N_{2}$ & $N_{3}$ & $N_{1} / N_{0}$ & $1 / p$ & $N_{2} / N_{0}$ & $1 / p^{2}$ & $N_{3} / N_{0}$ & $1 / p^{3}$ \\
\hline 3 & 2 & 1577 & 553 & 172 & 50 & 0.35 & 0.33 & 0.11 & 0.11 & 0.032 & 0.037 \\
5 & 2 & 1596 & 326 & 55 & 9 & 0.20 & 0.20 & 0.034 & 0.040 & 0.006 & 0.008 \\
5 & 4 & 1596 & 329 & 68 & 15 & 0.21 & 0.20 & 0.043 & 0.040 & 0.009 & 0.008 \\
5 & 3 & 2535 & 490 & 88 & 14 & 0.19 & 0.20 & 0.035 & 0.040 & 0.006 & 0.008 \\
7 & 3 & 1599 & 221 & 29 & & 0.14 & 0.14 & 0.018 & 0.020 & & \\
7 & 5 & 1599 & 256 & 39 & & 0.16 & 0.14 & 0.024 & 0.020 & &
\end{tabular}

If $t=1$, the situation is different. Indeed, by Eqs. (1)-(3) the constant term of $f(x, \chi \omega)$ equals

$$
a_{0}=(\chi(p)-1) B^{1}(\chi) ;
$$

hence, in the present case $\lambda_{\chi}$ is positive whenever $\chi(p)=\theta_{m}(p)=+1$. We must therefore modify the above hypothesis so as to concern those $f\left(x, \theta_{m} \omega\right)$ only for which $\theta_{m}(p)=-1$. We tested this hypothesis for $p=5, m>0$, obtaining the following ( $N_{k}^{\prime}$ denotes the number of $\lambda_{\chi} \geqslant k$ when $\theta_{m}(5)=-1$ ):

$$
N_{0}^{\prime}=1268, \quad N_{1}^{\prime}=241, \quad N_{2}^{\prime}=36 ; \quad N_{1}^{\prime} / N_{0}^{\prime}=0.19, \quad N_{2}^{\prime} / N_{0}^{\prime}=0.028 \text {. }
$$

We may also ask how often $\lambda^{-}$is, say, positive as $p$ is fixed and $|m|$ increases. If $p \leqslant 11$, then $\lambda_{0}^{-}=0$, and so $\lambda^{-}>0$ exactly when at least one of the $s=(p-1) / 2$ numbers $T_{0}$ corresponding to the characters $\theta_{m} \omega^{t-1}$ vanishes $\bmod p$. To avoid the exceptional case $t=1$, consider positive $m$ only. Then it is again natural to assume that the values of $T_{0}$ be randomly distributed $\bmod p$, and this implies that the proportion of the number of fields with $\lambda^{-}>0$ to the number of all fields should be about $\rho_{p}=1-\left(1-p^{-1}\right)^{s}$. Below is a comparison between the observed and expected values of this proportion:

\begin{tabular}{r|c|c}
\multicolumn{1}{c|}{$p$} & observed proportion & $\rho_{p}$ \\
\hline 5 & $587 / 1596=0.37$ & 0.36 \\
7 & $204 / 530=0.38$ & 0.37 \\
11 & $100 / 279=0.36$ & 0.38
\end{tabular}

A table including all the results of our computations has been deposited in the UMT file; see Review 29 in this issue.

8. Comparison with Previous Results. We next describe the contents of the previously published tables about $\lambda^{-}$. These tables were used by us to check our results.

Gold [3], [4] has computed, for $p=3,5,7,11$, the $\lambda$-invariant of the quadratic field with discriminant $-d<0$. His results in [4, Table 2] cover the range $0<d \leqslant 264$. They agree completely with ours, and so do also the additional results presented in [3, Tables 2 and 5] after the following apparent errors are corrected: In Table 2, the value 1253 for $d$ should be 1263 (corresponding to the given class number 20); in 
Table 5 , lines 5 and 6 , instead of $\lambda=3$ and $\lambda=4$ one should read $\lambda=2$. The latter correction is confirmed not only by [6] quoted below, but also by Corollary 5 in [3]. The expressions for $e_{n}$ in Table 5 should be correspondingly corrected.

Kobayashi [6] investigates, for $p=3$, the power series $f(x, \chi \omega)$ with $\chi=\theta_{m}$ and $\chi=\theta_{m} \omega$. He has determined the coefficients $a_{0}, \ldots, a_{8} \bmod 9$ of this power series for $-10^{4}<m<0$ and $0<3 m<10^{4}$. From his table one can read the value of $\lambda_{\chi}$, since in all cases $\lambda_{\chi} \leqslant 8$. Note that for $\chi=\theta_{m}$ the table is far more extensive than ours, while for $\chi=\theta_{m} \omega$ our computations go a bit farther. The overlapping parts of both tables are in agreement, except that the table in [6] omits the first negative $m$ with $\lambda_{\chi}>0$, namely $m=-2$. The nonvanishing of $\lambda_{x}$ in this case follows, by (11), from the fact that $\chi(3)=\theta_{-2}(3)=+1$. Our computation indeed shows, in agreement with [4], that $\lambda_{\chi}=1$.

The first author has determined, for $p<10^{4}$, the components $\lambda_{\chi}$ with $\chi=\theta_{m} \omega^{t \cdots 1}$ for $m=-1$ and $m=-3$ (see [2] and [1], respectively). For $t=3,5, \ldots, p-2$, one has in this range $\lambda_{\chi}=1$ if $(p, t-1)$ is an $E$-irregular or $D$-irregular pair, respectively, and $\lambda_{\chi}=0$ otherwise. A comparison of the tables in [1] and [2] with the present Table 2 shows no discrepancies.

The paper [5] by Hao and Parry tabulates the " $m$-irregular" primes $p<5025$ for the values of $m$ that appear in our Table 2. For a fixed $m$, the prime $p$ is $m$-irregular if and only if there is at least one $t>1$ such that $\lambda_{\chi}>0$ with $\chi=\theta_{m} \omega^{t-1}$. It is easily checked that, for $p<200$, the lists given in [5] coincide with the corresponding lists extracted from Table 2. Our computations show the somewhat interesting fact that every positive value of $\lambda_{\chi}$ in this region in fact equals 1 , except for a single value $\lambda_{\chi}=2$ occurring for $p=23$ and $\chi=\theta_{-2} \omega^{10}$.

Let us finally mention that if $m=-q$, with $q$ a prime, and $\theta_{m}(p)=-1$, then it follows from (11) that $\lambda_{\theta_{m}}>0$ exactly when the class number of the field $\mathbf{Q}(\sqrt{-q})$ is divisible by $p$. Thus a partial check of our results is also provided by the class number tables of imaginary quadratic fields.

9. The computations. The computations were run on the DEC-20 computer at the University of Turku. The programs, written in Fortran, used only integer arithmetic.

As is seen from Sections 5 and 6, the main task was the computing of the sums $S_{n k}$ (mostly for $n=1$ ). This was started by searching a primitive $\operatorname{root} \bmod p$ and constructing the index table. After decomposing $m$ into prime factors, the character values $\theta_{m}(a)$ were calculated via the Legendre symbol, using the congruence

$$
\left(\frac{a}{q}\right) \equiv a^{(q-1) / 2}(\bmod q) \quad(q \text { an odd prime factor of } m)
$$

and then checking that $\theta_{m}(a)$ indeed equals \pm 1 or 0 . For a fixed $t$, we chose a minimal $b>0$ such that $(b, d p)=1$ and $\theta_{m}(b) b^{t} \not \equiv 1(\bmod p)$. To find the value of $v(a)$ for $n=1$ (see (6) and (5)), we computed $a^{p-1} \bmod p^{2}$ by employing the 2-adic expansion of $p-1$ and the residues of $a^{2}, a^{4}, a^{8}, \ldots \bmod p^{2}$.

After computing the numbers $S_{1 k}$ mod $p$ we searched for the first nonvanishing number in the sequence $T_{0}^{(1)}, \ldots, T_{p-2}^{(1)} \bmod p$. The cases in which such a number did not exist were afterwards picked out by hand and dealt with on the level $n=2$. The procedure on this level was similar, except that this time the determination of $v(a)$ required computations $\bmod p^{3}$. 
APPENDIX

TABLE 1

The positive values of $\lambda_{\chi}$ for $p=5, \chi=\theta_{m} \omega^{t-1}(t=2$ or 4$)$ and $0<m \leqslant 3147$.

\begin{tabular}{|c|c|c|c|c|c|c|c|c|c|c|c|}
\hline $\mathrm{m}$ & $t$ & ${ }^{\lambda} x$ & $\mathrm{~m}$ & $t$ & $\lambda_{x}$ & $\mathrm{~m}$ & $t$ & $\lambda_{x}$ & $\mathrm{~m}$ & $t$ & $\lambda_{x}$ \\
\hline 14 & 2 & 1 & 267 & 2 & 2 & 509 & 2 & 2 & 734 & 2 & 1 \\
\hline 3 & 2 & 2 & 271 & 4 & 1 & 509 & 4 & 1 & 734 & 4. & 2 \\
\hline 26 & 2 & 1 & 278 & 4 & 3 & 514 & $i_{4}$ & 1 & 741 & 2 & 1 \\
\hline 31 & 2 & 1 & 201 & 2 & 1 & 519 & 4 & 1 & 743 & 2 & 1 \\
\hline 37 & 2 & 2 & 282 & 2 & 1 & 523 & 4 & 1. & 753 & 2 & 1 \\
\hline 30 & 4 & 1 & 287 & 4 & 1 & 526 & 4 & 1 & 753 & 4 & 2 \\
\hline 39 & 4 & 1 & 293 & 2 & 1 & 534 & 4 & 1 & 754 & 2 & 1 \\
\hline 42 & 4 & 1 & 290 & 2 & 1 & 537 & 4 & 1 & 758 & 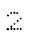 & 1 \\
\hline 51 & 4 & 1 & 293 & 4 & 1 & 541 & 4 & 1 & 759 & 2 & 1 \\
\hline 53 & 4 & 1 & 307 & 2 & 1 & 543 & 4 & 1 & 761 & 4 & 1 \\
\hline 59 & 2 & 1 & 313 & 2 & 2 & 554 & 2 & 1 & 763 & 2 & 1 \\
\hline 62 & 4 & 1 & 314 & 4 & 2 & $55 ?$ & 2 & 1 & 766 & 2 & 1 \\
\hline 69 & 4 & 1 & 326 & 4 & 1 & 574 & 2 & 1 & 767 & 4 & 1 \\
\hline 73 & 4 & 1 & 347 & 2 & 1 & $574_{r}$ & 4 & 1 & 781 & 2 & 1 \\
\hline 82 & 4 & 1 & 358 & 2 & 1 & 577 & 2 & 1 & 789 & 4 & 1 \\
\hline 86 & 2 & 1 & 366 & 4 & 1 & 581 & 2 & 1 & 791 & 4 & 1 \\
\hline 39 & 4 & 1 & 382 & $4_{4}$ & 1 & 531 & 4 & 2 & 794 & 2 & 1 \\
\hline 107 & $i_{4}$ & 1 & 391 & 2 & 1 & 587 & 2 & 1 & 790 & 4 & 1 \\
\hline 109 & 4 & 2 & 398 & 2 & 2 & 587 & 4 & 2 & 809 & 2 & 1 \\
\hline 114 & 4 & 3 & 401 & 4. & $\mathrm{i}$ & 589 & 4 & $j$ & 814 & 2 & 1 \\
\hline 123 & 2 & 1 & 407 & 4 & 1 & 591 & 2 & $\dot{1}$ & 217 & 4 & 1 \\
\hline 127 & 2 & $z$ & 422 & 4 & 1 & 597 & $4 \mathrm{r}$ & 2 & 822 & 2 & 1 \\
\hline 127 & 4 & 2 & 426 & 2 & 1 & 602 & 4 & 1 & 339 & 2 & 1 \\
\hline $12 \%$ & 2 & 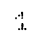 & 426 & 4 & $j$ & 606 & 4 & 1 & 842 & 2 & 1 \\
\hline 134 & 4 & 1 & 427 & 2 & 1 & 611 & 2 & 1 & $85 \%$ & 2 & 1 \\
\hline 139 & 4 & 1 & 427 & 4 & 1 & 617 & 4 & 1 & 857 & 4 & 1 \\
\hline 143 & 4 & 1 & 433 & 4 & 2 & 62 & 4 & 1 & 861 & 2 & 2 \\
\hline $1<9$ & 2 & 2 & 434 & $4 r$ & 2 & 623 & 4 & 2 & 869 & 4 & 2 \\
\hline 159 & 2 & 1 & 438 & 2 & 1 & 626 & 2 & 2 & 874 & 4 & 1 \\
\hline 161 & 4 & 1 & 439 & 4 & 4 & 626 & 4 & 1 & 881 & 2 & $\therefore$ \\
\hline 183 & 4 & 1 & 446 & 2 & 2 & 627 & 4 & 1 & 881 & 4 & 1 \\
\hline 156 & 4 & 1 & 4.53 & 4 & 2 & 629 & 2 & 2 & 887 & 2 & 1 \\
\hline 187 & 2 & 1 & 457 & 2 & 3 & 629 & 4 & 1 & 889 & 2 & $i$ \\
\hline 191 & 2 & 1 & 457 & 4 & 1 & 631 & 2 & 1 & 893 & 4 & 1 \\
\hline 191 & 4 & 1 & 458 & 2 & 1 & 633 & 2 & 3 & 903 & 2 & 1 \\
\hline 199 & 2 & 1 & 466 & 4 & 2 & 634 & 4 & 1 & 911 & 2 & 1 \\
\hline 20 & 4 & 1 & 467 & 2 & 1 & 643 & 2 & 1. & 917 & 2 & 1 \\
\hline 211 & 4 & 1 & 467 & 4 & 1 & 654 & $z$ & 1 & 921 & 2 & 1 \\
\hline 213 & 2 & 1 & 469 & 2 & 1 & 662 & 4 & 1 & 922 & 2 & 1 \\
\hline 214 & 4 & 1 & 471 & 2 & 2 & 673 & 4 & 1 & 922 & 4 & 1 \\
\hline 217 & 4 & 1 & 473 & 4 & 1 & 674 & 4 & 1 & 923 & 4 & 1 \\
\hline 22 & 2 & 1 & 479 & 2 & 1 & 678 & 2 & 1 & 926 & 2 & \pm \\
\hline 23 & 2 & 1 & 489 & 4 & 1 & 679 & 2 & 1 & 933 & 4 & 1 \\
\hline 227 & 2 & 1 & 497 & 4 & 1 & 681 & 2 & 1 & 937 & 2 & 1 \\
\hline 237 & 2 & 1 & 498 & 2 & 2 & 683 & 2 & 1 & 939 & 2 & 1 \\
\hline 238 & 4 & 1 & 499 & 2 & 1 & 687 & 4 & 1 & 943 & 4 & 1 \\
\hline 241 & 4 & 1 & 499 & 4 & 1 & 689 & 2 & 1 & 946 & 2 & 1 \\
\hline 253 & 2 & 2 & 501 & 2 & 1 & 699 & 4 & 1 & 947 & 4 & 1 \\
\hline 257 & 2 & 1 & 501 & 4 & 1 & 717 & 2 & $j$ & 949 & 2 & 1 \\
\hline 257 & 4 & 1 & 502 & 2 & 1 & 719 & 4 & 2 & 957 & 2 & 1 \\
\hline 259 & 4 & 1 & 502 & 4 & 1 & 727 & 4. & 1 & 966 & $i_{\mathrm{r}}$ & 1 \\
\hline
\end{tabular}


TABLE 1 ( continued)

\begin{tabular}{|c|c|c|c|c|c|c|c|c|c|c|c|}
\hline $\mathrm{m}$ & $t$ & ${ }^{\lambda} x$ & $\mathrm{~m}$ & $t$ & ${ }^{\lambda}{ }_{x}$ & $\mathrm{~m}$ & $t$ & ${ }^{\lambda} x$ & $\mathrm{~m}$ & $t$ & $\lambda_{X}$ \\
\hline 973 & 2 & 1 & 1214 & 4 & 1 & 1483 & 2 & 1 & 1797 & 4 & 2 \\
\hline 978 & 4 & 1 & 1217 & 2 & 1 & 1486 & 4 & 1 & 1798 & 4 & 1 \\
\hline 982 & 4 & 1 & 1226 & 4 & 1 & 1493 & 2 & 1 & 1799 & 2 & 1 \\
\hline 983 & 4 & 1 & 1231 & 2 & 3 & 1493 & 4 & 1 & 1803 & 4 & 1 \\
\hline 986 & 2 & 1 & 1231 & 4 & 1 & 1506 & 2 & 1 & 1817 & 4 & 2 \\
\hline 997 & 2 & 1 & 1238 & 2 & 1 & 1509 & 2 & 1 & 1829 & 2 & 1 \\
\hline 1003 & 2 & 1 & 1238 & 4 & 1 & 1511 & 2 & 1 & 1829 & 4 & 1 \\
\hline 1006 & 2 & 2 & 1243 & 4 & 1 & 1514 & 2 & 1 & 1.934 & 2 & 1 \\
\hline $100 \%$ & 2 & 1 & 1247 & 4 & 2 & 1510 & 2 & 1 & 1834 & 4 & 1 \\
\hline 1018 & 2 & 1 & 1253 & 2 & 1 & 1518 & 4 & 3 & 1837 & 2 & 2 \\
\hline 1031 & 4 & 1 & 1254 & 2 & 1 & 1529 & 4 & 2 & 1838 & $\therefore$ & 1. \\
\hline 1034 & 2 & 1 & 1261 & 4 & 1 & 1531 & 2 & 1 & 1846 & 2 & 1 \\
\hline 1037 & 2 & 1 & 1262 & 4 & 1 & 1531 & 4 & 1 & 1847 & 2 & 1 \\
\hline 1041 & 4 & 1 & 1267 & 4 & 1 & 1533 & 2 & 1 & 1851 & 4 & 1 \\
\hline 1042 & 4 & 1 & 1273 & 2 & 1 & $154 \%$ & 2 & 1 & 1853 & 2 & $\mathrm{j}$ \\
\hline 1051 & 4 & 1 & 1279 & 2 & 1 & 1546 & 2 & 1 & 1853 & 4 & 1 \\
\hline 1059 & 2 & 1 & 1279 & 4. & 1 & 1571 & 2 & 1 & 1861 & 4 & 1 \\
\hline 1063 & 2 & 1 & 1281 & 4 & 1 & 1577 & 4 & 1 & 1874 & 4 & 1 \\
\hline 1069 & 2 & 1 & 1289 & 4. & 1 & 1579 & 2 & 1 & 1882 & 2 & 1 \\
\hline 1073 & 2 & 3 & 1291 & 2 & 1 & 1582 & 2 & 1 & 1891 & 4 & 1 \\
\hline 1074 & 4 & 1 & 1293 & 4 & 1 & 1586 & 4 & 1 & 1897 & 4 & 3 \\
\hline 1077 & 2 & 1 & 1294 & 2 & 1 & 1597 & 2 & 1 & 1898 & 4 & 1 \\
\hline 1079 & 4 & 2 & 1301 & 4 & 1 & 1597 & 4 & 1 & 1907 & 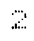 & 1 \\
\hline 1086 & 4 & 2 & 1313 & 4 & 2 & 1621 & 4 & 1 & 1907 & 4 & 1. \\
\hline 1087 & 4 & 1 & 1317 & 4 & 3 & 1631 & 2 & 1 & 1913 & 2 & 1 \\
\hline 1093 & 4 & 1 & 1321 & 4 & 2 & 1631 & $4_{1}^{4}$ & 1 & 1913 & 4 & 1 \\
\hline 1097 & 2 & 1 & 1327 & 2 & 1 & 1633 & 4 & 2 & 1914 & 2 & 1 \\
\hline 1106 & 2 & 1 & 1327 & 4 & 1 & 1637 & 4 & 1 & 1914 & 4 & 1 \\
\hline 1111 & 4 & 2 & 1338 & 4 & 1 & 1641 & 4 & 1 & 1921 & 4 & 1 \\
\hline 1113 & 2 & 1 & 1339 & 4 & 1 & 1654 & 2 & 1 & 1923 & 2 & 1. \\
\hline 1113 & 4 & 1 & 1342 & 2 & 1 & 1658 & 4 & 1 & 1934 & 2 & 1. \\
\hline 1114 & 2 & 1 & 1351 & 4 & 1 & 1662 & 4 & 2 & 1937 & 2 & 1 \\
\hline 1118 & 4. & 1 & 1354 & 2 & 1 & 1663 & 4 & 1 & 1938 & 2 & 1 \\
\hline 1119 & 2 & 1 & 1366 & 2 & 1 & 1686 & 4 & 1. & 1938 & 4 & 1 \\
\hline 1121 & 2 & 1 & 1366 & 4 & 1 & 1699 & 4 & 1 & 1941 & 2 & 1 \\
\hline 112 & 2 & 1 & 1379 & 2 & 1 & 1702 & 4 & 2 & 1943 & 4 & 3 \\
\hline 1123 & 2 & 1 & 1382 & 4 & 1 & 1713 & 4 & 1 & 1949 & 2 & 2 \\
\hline 1126 & 2 & 1 & 1389 & 2 & 1 & 1717 & 4 & 1 & 1954 & 2 & 1 \\
\hline 1126 & 4 & 1 & 1389 & 4 & 1 & 1721 & 2 & 1 & 1957 & 2 & 1 \\
\hline 1129 & 2 & 1 & 1393 & 4 & 2 & 1723 & 4 & 1 & 1959 & 4 & 1 \\
\hline 1131 & 2 & 2 & 1398 & 4 & 1 & 1731 & 4 & 1 & 1960 & 4 & 1. \\
\hline 1133 & 4 & 1 & 1401 & 2 & 1 & 1738 & 2 & 1 & 1969 & $i_{4}$ & 1. \\
\hline 1137 & 2 & 1 & 1402 & 4 & 2 & 1733 & 4 & 2 & 1973 & 2 & 1 \\
\hline 1137 & 4 & 1 & 1406 & 4 & 1 & 1739 & 2 & 1 & 1977 & 2 & 1 \\
\hline 1142 & 2 & 1 & 1407 & 4. & 1 & 1741 & 4 & 1 & 1979 & 2 & 1 \\
\hline 1149 & 2 & 1 & 1426 & 4 & 1 & 1754 & 4 & 1 & 1982 & 4 & 1 \\
\hline 1157 & 4 & 1 & 1427 & 4 & $i_{+}$ & 1757 & 2 & 1 & 1986 & 4 & 1 \\
\hline $116 ?$ & 2 & 1 & 1429 & 4. & 1 & 1758 & 2 & 1. & 1999 & 2 & 1 \\
\hline 1173 & 4 & 1 & 1434 & 2 & 2 & 1758 & 4 & 1 & 1999 & $\alpha_{4}$ & 1 \\
\hline 1182 & 4 & 2 & $143^{4}$ & 4 & 1 & 1761 & 4 & 2 & 2001 & 2 & 1 \\
\hline 1187 & 4 & 1 & 1441 & 4 & 1 & 1762 & 4 & 1 & 2002 & 2 & 1 \\
\hline 1191 & 4 & 1 & 1443 & 4 & 3 & 1760 & 4 & 1 & 2002 & 4 & 1 \\
\hline 1193 & 2 & 2 & 1451 & 2 & $\dot{I}$ & 1769 & 2 & 1 & 2003 & 2 & 1 \\
\hline 1194 & 4 & 1 & 1464 & 4. & 1 & 1777 & $i_{4}$ & 1 & 2003 & 4 & 3 \\
\hline 1198 & 2 & 1 & 1466 & 2 & 2 & 1779 & 2 & 1 & 2014 & $i_{4}$ & 1 \\
\hline 1203 & 2 & 1 & 1478 & 2 & 3 & 1783 & 2 & 1. & 2027 & 2 & 1. \\
\hline 1213 & 4 & 1 & 1479 & 4 & 2 & 1756 & 4 & 1 & $202 \%$ & ; & 1 \\
\hline
\end{tabular}


TABLE 1 (continued)

\begin{tabular}{|c|c|c|c|c|c|c|c|c|c|c|c|}
\hline $\mathrm{m}$ & $t$ & $\lambda_{x}$ & $\mathrm{~m}$ & $t$ & $\lambda_{x}$ & $\mathrm{~m}$ & $t$ & ${ }^{\lambda} x$ & $\mathrm{~m}$ & $t$ & ${ }^{\lambda}{ }_{x}$ \\
\hline 2029 & 2 & 2 & 2307 & 2 & 1 & 2571 & 4 & 1 & 2878 & 4 & 1 \\
\hline 2031 & 4 & 1 & 2307 & 4 & 1 & 2573 & $\therefore$ & 1 & 2882 & 2 & 2 \\
\hline 2033 & 4 & 1 & 2314 & 2 & 1 & 2573 & 4 & 1 & 2886 & 4 & 1 \\
\hline 2038 & 4 & 1 & 2317 & 2 & 1 & 2577 & 4. & 1 & 2887 & 2 & 1 \\
\hline 2039 & 2 & 1. & 2323 & 4 & 3 & 2570 & 4 & 1 & 2911 & 4. & 1 \\
\hline 2051 & 2 & 2 & 2326 & 4 & 1 & 2579 & 4. & 1 & 2914 & 2 & 2 \\
\hline 2081 & 4 & 1 & 2329 & 2 & 1 & 2581 & 2 & 1 & 2923 & 4 & 1 \\
\hline 2083 & 2 & 1 & 2333 & 2 & 1 & 2599 & 4 & 1 & 2927 & 2 & 1 \\
\hline 2033 & 4 & 1 & 2334 & $4_{4}$ & 1 & 2602 & 4 & 1 & 2931 & 4 & 1 \\
\hline 2087 & 2 & 1 & 2341 & 2 & 1 & 2603 & 2 & 1 & 2946 & 2 & 1 \\
\hline 2009 & 3 & 1 & 2342 & 4 & 1 & 2609 & 4 & 2 & 2949 & 2 & 1 \\
\hline 2093 & 2 & 1. & 2347 & $z$ & 1 & 2633 & 2 & 1 & 2959 & 2 & $\dot{1}$ \\
\hline 2098 & 2 & 1 & 2353 & 2 & 2 & 2634 & 4 & 1 & 2963 & 2 & 2 \\
\hline 2101 & 4 & 2 & 2354 & 2 & 1. & 2639 & 2 & 1 & 2966 & 4 & 1 \\
\hline 202 & 2 & 1 & 2359 & 4 & 1 & 2647 & 2 & 1. & 2967 & 2 & 2 \\
\hline 2111 & 4 & 2 & 2362 & 4 & 1 & 2654 & 4 & 2 & 2971 & 2 & 1 \\
\hline 2149 & 4 & $i$ & 2373 & 2 & 2 & 2657 & 4 & 1 & 2973 & 4 & 1 \\
\hline 2122 & 4 & 2 & 2381 & 2 & 1 & 2661 & $\therefore$ & 1 & 2974 & 2 & 2 \\
\hline 2123 & 2 & 1 & 2386 & 2 & 1 & 2667 & 4 & 2 & 2983 & 4 & 2 \\
\hline 2126 & 2 & 1. & 2386 & 4 & 1 & 2669 & 2 & 1 & 2986 & 4. & 2 \\
\hline 2127 & 2 & 1 & 2391 & 2 & 1 & 2671 & 2 & 1 & 2991 & 2 & 1 \\
\hline 2129 & 2 & 1 & 2391 & 4 & 2 & 2671 & 4 & 1 & 2991 & 4 & 1 \\
\hline 2131 & 2 & 1 & 2397 & 4 & 1 & 2633 & 4 & 3 & 2993 & 4 & 1 \\
\hline $2+43$ & 2 & 1 & 2399 & 4. & 1 & 2686 & 2 & 1 & 2994 & $i_{4}$ & 1 \\
\hline 2146 & 2 & 1. & 2406 & 4 & 1 & 2687 & 2 & 1 & 2998 & 2 & 1 \\
\hline 2153 & 2 & 1 & 2411 & 4 & 1 & 2687 & 4 & 1 & 3013 & 2 & 1 \\
\hline 2153 & 4 & 1 & 2433 & 2 & 2 & 2694 & 4 & 1 & 3014 & 2 & 2 \\
\hline 2457 & 4 & 1 & 2438 & 2 & 2 & 2698 & $\therefore$ & 2 & 3023 & 2 & 1 \\
\hline 2158 & 4 & 1 & 2438 & $i_{4}$ & 1 & 2706 & 2 & 1 & 3039 & 4 & 1. \\
\hline 2159 & 2 & 1 & 2446 & 2 & 1 & 2711 & 2 & 1. & 3041 & 4 & 1 \\
\hline 2161 & 2 & 2 & 2449 & 2 & 1 & 2714 & 2 & 3 & 3053 & 2 & 1 \\
\hline 2171 & 4 & 1 & 2459 & 4 & 2 & 272 & 2 & 2 & 3054 & 4 & 1 \\
\hline 2177 & 2 & 2 & 2462 & 2 & 2 & 2723 & $z$ & 3 & 3059 & 4 & 2 \\
\hline 2181 & 2 & 1 & 2471 & 4 & 2 & 2723 & 4 & 2 & 3077 & 4 & 1 \\
\hline 2182 & 2 & 1 & 2431 & 2 & 2 & 2731 & 4 & 1 & 3082 & 4 & 1 \\
\hline 2189 & 2 & $\mathrm{i}$ & 2482 & 2 & 1 & 2739 & 4 & 2 & 3098 & 2 & 1. \\
\hline 2139 & 4 & \pm & 2486 & 2 & 1. & 2741 & 4 & 2 & 3101 & 4 & 2 \\
\hline 2194 & 2 & 1. & 2487 & 2 & 1 & 2742 & 2 & 1 & 3102 & 2 & 1 \\
\hline 219 & 2 & 1 & 2439 & 2 & 3 & 2743 & 4 & 2 & 3103 & 2 & 1 \\
\hline 221 & 2 & 1 & 2498 & 4 & 3 & 2746 & 4. & 1 & 3106 & 2 & 2 \\
\hline 222 & 2 & 1 & 2501 & 4 & 2 & 2759 & 2 & 2 & 3107 & 2 & 1. \\
\hline 229 & 2 & 1 & 2503 & 2 & 1 & 2766 & 2 & 1 & 311 & 4 & 1 \\
\hline 231 & 2 & 3 & 2503 & 4 & 1 & 2771 & 2 & 1 & 3113 & 2 & 1 \\
\hline 2234 & 4 & 3 & 2509 & 2 & 1 & 2773 & $\ddot{z}$ & 1 & 3121 & 2 & 1 \\
\hline 243 & 2 & 1 & 2513 & 4 & 1 & 2778 & 2 & 1 & 3121 & 4 & 1 \\
\hline 2243 & 4 & 1 & 2519 & 2 & 1. & 2803 & 4 & 1 & 3126 & 2 & 1 \\
\hline 2257 & 4 & 1 & 2522 & 4 & 2 & 2814 & 2 & 1 & 3127 & 2 & 1 \\
\hline 262 & 4 & 1 & 2526 & 2 & 1 & 2021 & 2 & 1 & 3129 & 2 & 1 \\
\hline 263 & 2 & 2 & 2531 & 2 & 1. & 2322 & 4 & 1 & 3129 & 4 & 1 \\
\hline 2263 & 4 & 1 & 2533 & 4 & 1. & 2829 & 2 & 1 & 3134 & 4 & 1. \\
\hline 271 & 2 & 1. & 2534 & 2 & 2 & 2829 & 4 & 3 & $\$ 138$ & 4 & 1 \\
\hline 2273 & 2 & $\dot{1}$ & 2546 & 4 & 1 & 2841 & 2 & 1 & 3147 & 2 & 1 \\
\hline 2278 & 2 & 2 & 2551 & 2 & 1 & 2043 & 4 & 1 & & & \\
\hline 2279 & 4 & 1 & 2557 & 4 & 1 & 2851 & 4 & 1 & & & \\
\hline 281 & 2 & 2 & 2558 & 4 & 1 & 2559 & 2 & 1 & & & \\
\hline 287 & 4 & 1 & 2563 & 2 & 1 & 2877 & 4 & 1 & & & \\
\hline 2306 & $z$ & 1 & 2566 & 4 & 1 & 2878 & 2 & 2 & & & \\
\hline
\end{tabular}


TABLE 2

The values of $t, 1 \leqslant t \leqslant p-1$, for which $\lambda_{\chi}>0$ with $\chi=\theta_{m} \omega^{t-1}$, in the region $2<p<200, m=-7,-3,-2,-1,2,5$. The dagger $\left({ }^{\dagger}\right)$ indicates that $\lambda_{x}=2$; in all other cases $\lambda_{x}=1$.

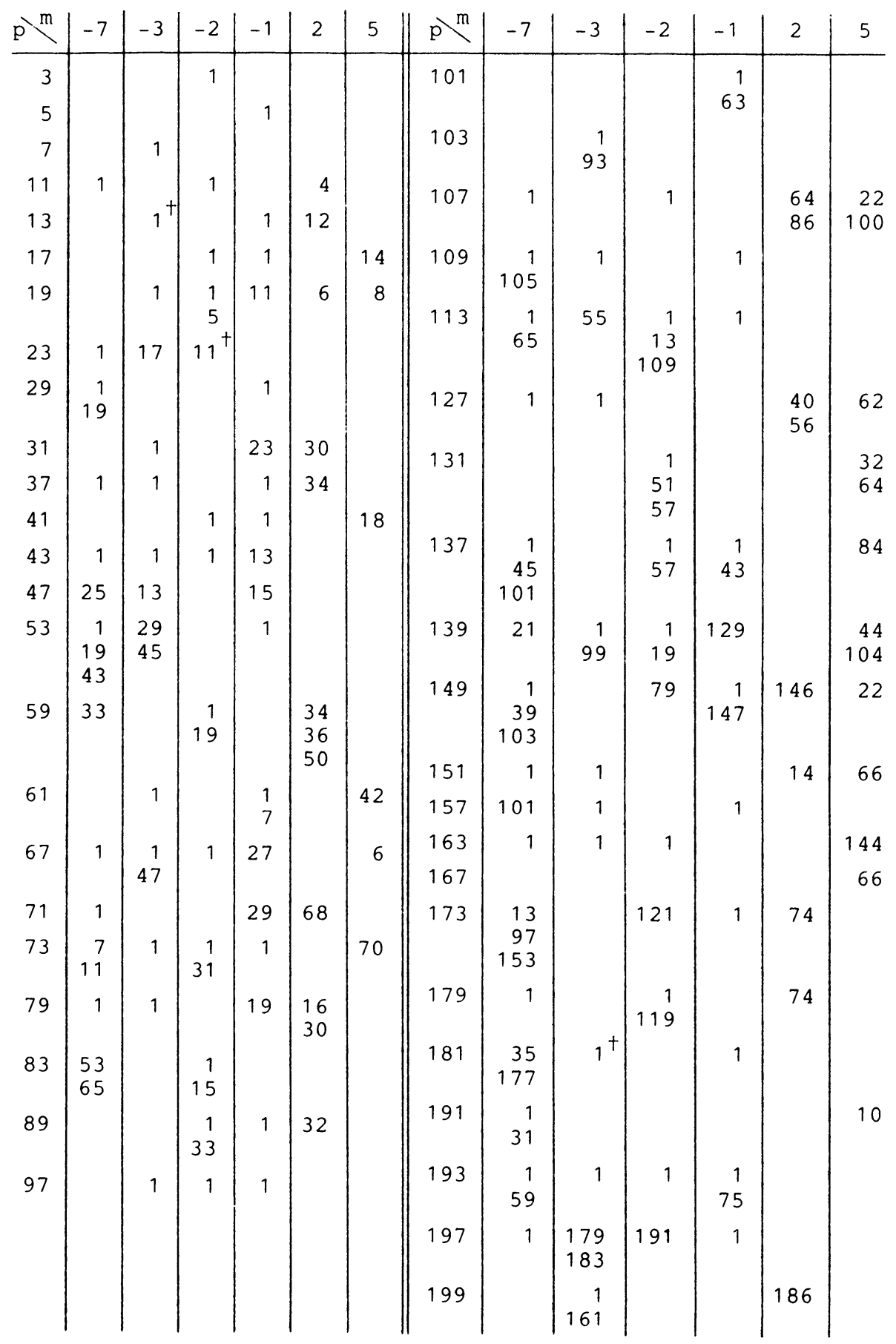


Department of Mathematics

University of Turku

SF-20500 Turku, Finland

1. R. Ernvall, "Generalized Bernoulli numbers, generalized irregular primes, and class number," Ann. Univ. Turku. Ser. A I, No. 178, 1979, 72 pp.

2. R. ERnvall \& T. Metsänkylä, "Cyclotomic invariants and E-irregular primes," Math. Comp., v. 32, 1978, pp. 617-629.

3. R. Gold, “Examples of Iwasawa invariants," Acta Arith. v. 26, 1974, pp. 21-32.

4. R. Gold, “Examples of Iwasawa invariants, II," Acta Arith., v. 26, 1975, pp. 232-240.

5. F. H. HAO \& C. J. PARRY, "Generalized Bernoulli numbers and $m$-regular primes," Math. Comp., v. 43,1984 , pp. $273-288$.

6. S. Kobayashi, "Calcul approché de la série d'Iwasawa pour les corps quadratiques $(p=3)$," Number Theory, 1981-82 and 1982-83, Exp. No. 4, 68 pp., Publ. Math. Fac. Sci. Besançon, Univ. Franche-Comté, Besançon, 1983.

7. H. W. LEOPOLDT, "Eine Verallgemeinerung der Bernoullischen Zahlen," Abh. Math. Sem. Univ. Hamburg, v. 22, 1958, pp. 131-140.

8. T. MetsäNKYLÄ, "Iwasawa invariants and Kummer congruences," J. Number Theory, v. 10, 1978, pp. 510-522.

9. I. Sh. Slavutskil, "Local properties of Bernoulli numbers and a generalization of the KummerVandiver theorem," Izv. Vyssh. Uchebn. Zaved. Mat., No. 3 (118), 1972, pp. 61-69. (Russian)

10. S. S. Wagstaff, JR., “The irregular primes to 125000," Math. Comp., v. 32, 1978, pp. 583-591.

11. L. C. Washington, Introduction to Cyclotomic Fields, Springer-Verlag, Berlin and New York, 1982. 